PROCEEDINGS OF THE

AMERICAN MATHEMATICAL SOCIETY

Volume 138, Number 9, September 2010, Pages 3125-3133

S 0002-9939(10)10342-6

Article electronically published on April 6, 2010

\title{
SYMMETRIC UTUMI QUOTIENT RINGS OF ORE EXTENSIONS BY SKEW DERIVATIONS
}

\author{
CHEN-LIAN CHUANG AND YUAN-TSUNG TSAI
}

(Communicated by Gail R. Letzter)

\begin{abstract}
Let $R$ be a ring, $X$ a sequence of noncommuting indeterminates $x_{1}, x_{2}, \ldots$ and $D$ a sequence of skew derivations $\delta_{1}, \delta_{2}, \ldots$, where each $\delta_{i}$ is a $\sigma_{i}$-derivation of $R$. The Ore extension of $R$ by $D$, denoted by $R[X ; D]$, is the ring generated by $R$ and $X$ subjected to the rule $x_{i} r=\sigma_{i}(r) x_{i}+\delta_{i}(r)$ for each $i$. If $|X| \geq 2$ and $R$ is a domain, we show that the symmetric maximal ring of quotients of $R[X ; D]$ is equal to $U_{s}(R)[X ; D]$, where $U_{s}(R)$ is the symmetric maximal ring of quotients of $R$.
\end{abstract}

\section{INTRODUCTION AND RESULTS}

Rings here are always associative but may not have the multiplication identity. By a $\sigma$-derivation of a ring $R$, where $\sigma$ is an automorphism of $R$, we mean a map $\delta: R \rightarrow R$ satisfying

$$
\delta(a+b)=\delta(a)+\delta(b), \quad \delta(a b)=\delta(a) b+\sigma(a) \delta(b) \quad \text { for } a, b \in R .
$$

We call $\sigma$ the associated automorphism of $\delta$. Let $\mathcal{A}$ be the set of all automorphisms of $R$. For $\sigma \in \mathcal{A}$, let $\mathcal{L}_{\sigma}$ be the set of all $\sigma$-derivations of $R$. Set $\mathcal{L}=\bigcup_{\sigma \in \mathcal{A}} \mathcal{L}_{\sigma}$. Elements of $\mathcal{L}$ are generally called skew derivations.

Let $X$ be a set of noncommuting indeterminates. Given a map $\phi: X \rightarrow \mathcal{L}$, let $R[X ; \phi]$ denote the ring of polynomials in noncommuting indeterminates $x \in X$ and with coefficients in $R$ subjected to the following commutation rule for $a \in R$ and $x \in X$ :

$$
x a=\sigma(a) x+\delta(a), \text { where } \delta=\phi(x) \text { is a } \sigma \text {-derivation. }
$$

We call $R[X ; \phi]$ the Ore extension of $R$ by $\phi$. (See [4, 6, 25, 26].) We stress here that the indeterminates $x \in X$ do not commute with each other. The map $\phi$ may not be injective. So distinct $x \in X$ can be associated with the same skew derivation. In traditional notation, we denote $\phi(x)$ by $\delta_{x}$, its associated automorphism by $\sigma_{x}$ and then $R[X ; \phi]$ by $R\left[x ; \sigma_{x}, \delta_{x}\right]_{x \in X}$. Alternatively, we can also enumerate $X$ as a (finite or transfinite) sequence $x_{i}, i=0,1, \ldots$, and let $D$ be the corresponding sequence $\delta_{i}, i=0,1, \ldots$, where $\delta_{i} \stackrel{\text { def. }}{=} \phi\left(x_{i}\right)=\delta_{x_{i}} \in \mathcal{L}_{\sigma_{i}}$ and $\sigma_{i} \stackrel{\text { def. }}{=} \sigma_{x_{i}}$ is its associated automorphism. In this way, the map $\phi$ is explicitly encoded in the two corresponding sequences $X$ and $D$. We can thus denote $R[X ; \phi]$ by $R[X ; D]$. If

Received by the editors September 17, 2009 and, in revised form, December 16, 2009.

2010 Mathematics Subject Classification. Primary 16S36, 16S85, 16W25.

Key words and phrases. Domain, skew derivations, Ore extension, skew polynomial ring, symmetric Utumi quotient ring, symmetric maximal ring of quotients.

(C)2010 American Mathematical Society 3125

Reverts to public domain 28 years from publication 
$X$ is a singleton, say $X=\{x\}$, then $R[X ; D]$ is commonly written as $R[x ; \sigma, \delta]$, where $\delta=\phi(x)$ is a $\sigma$-derivation. If $\delta=0$, then $R[x ; \sigma, \delta]$ is abbreviated as $R[x ; \sigma]$, called the Ore extension of automorphism type. If $\sigma=1, R[x ; \sigma, \delta]$ is abbreviated as $R[x ; \delta]$, called the Ore extension of derivation type. Such Ore extensions have been most intensively investigated. As another interesting special case, if $\sigma_{i}=1$ and $\delta_{i}=0$ for all $i$, then $R[X ; D]$ is written as $R\langle X\rangle$, called the free ring generated by $X$ over $R$. Ore extensions are also called skew polynomial rings, which has become one of the most basic and useful constructions in ring theory. This topic has been extensively studied in various directions for a few decades. See, for example, [4, 5, 6, 7, 10, 11, 12, 13, 16, 17, 25, 26.

Let $U_{\ell}(R)$ (resp. $U_{r}(R)$ and $\left.U_{s}(R)\right)$ denote the left (resp. right and symmetric) Utumi quotient rings of a ring $R$. Utumi quotient rings are also called maximal rings of quotients. Our aim is the following.

Theorem 1. Let $R$ be a domain. If $X$ has cardinality $\geq 2$, then

$$
U_{s}(R[X ; D])=U_{s}(R)[X ; D] .
$$

The center of $U_{s}(R)$ is called the extended centroid of $R$. We have the following immediate corollary.

Corollary 2. Let $R$ be a domain with the extended centroid $C$. If $|X| \geq 2$, then the extended centroid of $R[X ; D]$ is equal to

$$
\left\{\alpha \in C: \delta_{x}(\alpha)=0 \text { and } \sigma_{x}(\alpha)=\alpha \text { for } x \in X\right\} .
$$

There are a lot of works that have been devoted to computing quotient rings; see for example, $8,2,18,19,24,25$. The special case of our result when $D$ consists of only ordinary derivations is proved in [25. If all these ordinary derivations vanish and if $R$ happens to be a field, then $R[X ; D]$ is merely the free algebra $R\langle X\rangle$ over the field $R$ and is shown in [1 to coincide with its symmetric Utumi quotient ring. Our work is strongly motivated by [1]. Actually, one of our crucial steps, namely Lemma 5. is based on the smart argument of [1. Other results in this direction can be found in $3,21,22,23,25,26$. We remark that the results here are false if $|X|=1$.

\section{Preliminaries}

We prove here an important discovery by D. Passman (Theorem 44), which gives half of our result. Meanwhile we review some basic notions about Utumi quotient rings. Rings in this section are arbitrary, not necessarily domains.

By a word, we mean a product (or monomial) $w=y_{1} y_{2} \cdots y_{n}$, where $y_{i} \in X$. We call $n$ the length of $w$ and denote it by $\operatorname{lh}(w)$. We also let the unit 1 denote the empty word with length 0 . With the commutation rule, any $0 \neq f \in R[X ; D]$ can be written in the form

$$
f=a_{0} w_{0}+a_{1} w_{1}+\cdots,
$$

where $a_{i} \in R \backslash\{0\}$ and where $w_{i}$ are distinct words in $X$. We call (1) the regular expression of $f$. Given $\rho \subseteq R$, we let $\rho[X ; D]$ denote the set of $f$ above with all $a_{i} \in \rho$.

We write $\rho \triangleleft_{r t} R$ to denote that $\rho$ is a right ideal of $R$. Given $\rho \triangleleft_{r t} R$ and $a \in R$, define $a^{-1} \rho \stackrel{\text { def. }}{=}\{r \in R: a r \in \rho\}$. A right ideal $\rho$ of $R$ is called dense if for any $a, b \in R, a\left(b^{-1} \rho\right)=0$ implies $a=0$, or equivalently, if given $0 \neq a, b \in R$, there 
exists $r \in R$ such that $a r \neq 0$ and $b r \in \rho$. We write $\rho \boldsymbol{\iota}_{r t} R$ to denote that $\rho$ is a right dense ideal of $R$. Left ideals and left dense ideals are defined analogously.

Lemma 3. If $\rho \boldsymbol{\triangleleft}_{r t} R$, then $\rho[X ; D] \boldsymbol{\iota}_{r t} R[X ; D]$.

Proof. Given $\rho \triangleleft_{r t} R$ and $\delta \in \mathcal{L}_{\sigma}$, define

$$
\rho_{\delta} \stackrel{\text { def. }}{=}\{r \in R: \delta(r) \in \rho \text { and } \sigma(r) \in \rho\} .
$$

We check easily that $\rho_{\delta} \triangleleft_{r t} R$. We claim that if $\rho \boldsymbol{\triangleleft}_{r t} R$, then $\rho_{\delta} \boldsymbol{\triangleleft}_{r t} R$. Reason. Given $b \in R, y \in b^{-1} \sigma^{-1}(\rho)$ and $z \in \delta(b y)^{-1} \rho$, we have $\sigma(b y), \delta(b y) z \in \rho$ and hence $\delta(b y z)=\delta(b y) z+\sigma(b y) \delta(z) \in \rho$. So byz $\in \rho_{\delta}$, that is, $y z \in b^{-1} \rho_{\delta}$. For $a \in R$, if $a\left(b^{-1} \rho_{\delta}\right)=0$, then $a y z=0$. Since $z \in \delta(b y)^{-1} \rho$ is arbitrary, we have $a y\left(\delta(b y)^{-1} \rho\right)=0$ and hence $a y=0$ by the right density of $\rho$. Since $y \in b^{-1} \sigma^{-1}(\rho)$ is arbitrary, we have $a\left(b^{-1} \sigma^{-1}(\rho)\right)=0$ and hence $a=0$ by the right density of $\sigma^{-1}(\rho)$. The claim is thus proved.

Returning to the Ore extension $R[X ; D]$, if $\phi(x)=\delta$, then

$$
x \rho_{\delta} \subseteq \sigma\left(\rho_{\delta}\right) x+\delta\left(\rho_{\delta}\right) \subseteq \rho x+\rho \subseteq \rho[X ; D] .
$$

Given a monomial $M=a x_{1} \cdots x_{n}$, where $a \in R$ and $x_{i} \in X$, define $\rho_{M}=a^{-1} \rho$ for $n=0$, and inductively for $n \geq 1$,

$$
\rho_{M} \stackrel{\text { def. }}{=}\left(\rho_{a x_{1} \cdots x_{n-1}}\right)_{\delta_{n}}, \quad \text { where } \delta_{n}=\phi\left(x_{n}\right) .
$$

Inductively, $M \rho_{M} \subseteq \rho[X ; D]$ and, by the claim above, if $\rho \boldsymbol{\triangleleft}_{r t} R$, then $\rho_{M} \boldsymbol{\triangleleft}_{r t} R$.

Now, assume $\rho \triangleleft_{r t} R$. Clearly, $\rho[X ; D] \triangleleft_{r t} R[X ; D]$. We show its density. Given $g \in R[X ; D]$, write $g=\sum_{i} M_{i}$, where $M_{i}$ are nonzero monomials with distinct words. Set $\rho^{\prime}=\bigcap_{i} \rho_{M_{i}}$. So $\rho^{\prime} \boldsymbol{\iota}_{r t} R$. Then $g \rho^{\prime} \subseteq \rho[X ; D]$, that is, $\rho^{\prime} \subseteq g^{-1} \rho[X ; D]$. If $0 \neq f \in R[X ; D]$, then $0 \neq f \rho^{\prime} \subseteq f\left(g^{-1} \rho[X ; D]\right)$, as asserted.

Unlike automorphisms or ordinary derivations, $\delta \in \mathcal{L}_{\sigma}$ for $\sigma \neq 1$ is not left-right symmetry, for in the expansion $\delta(a b)=\delta(a) b+\sigma(a) \delta(b)$, the associated automorphism $\sigma$ applies to the left factor $a$, not to the right factor $b$. However, there is a left version of Lemma 3 as follows: With the commutation rule, any $f \in R[X ; D]$ can also be brought to the form

$$
f=w_{0} b_{0}+w_{1} b_{1}+\cdots,
$$

where $b_{i} \in R \backslash\{0\}$ and where $w_{i}$ are distinct words in $X$. The left version of Lemma 3 says that if $\lambda$ is a left dense ideal of $R$, then so is $[X ; D] \lambda$, where $[X ; D] \lambda$ is the set of $f$ in $\left(1^{\prime}\right)$ with all $b_{i} \in \lambda$. To see this, we consider the following more general notion: By a $(\sigma, \tau)$-derivation of $R$, where $\sigma, \tau \in \mathcal{A}$, we mean a map $\delta: R \rightarrow R$ such that

$$
\delta(a+b)=\delta(a)+\delta(b), \quad \delta(a b)=\delta(a) \tau(b)+\sigma(a) \delta(b) \quad \text { for } a, b \in R .
$$

So $\sigma$-derivations are merely $(\sigma, 1)$-derivations. Let us also call $\tau$ the associated automorphism of a $(1, \tau)$-derivation. Given $\delta \in \mathcal{L}_{\sigma}, \tilde{\delta} \stackrel{\text { def. }}{=} \delta \sigma^{-1}$ is a $\left(1, \sigma^{-1}\right)$ derivation, as can be seen easily. Define the map $\tilde{\phi}$ by setting $\tilde{\phi}(x) \stackrel{\text { def. }}{=} \tilde{\delta}$ for $x \in X$. Given $x \in X$ such that $\delta \stackrel{\text { def. }}{=} \phi(x) \in \mathcal{L}_{\sigma}$, the commutation rule $x r=\sigma(r) x+\delta(r)$ for $r \in R$ can be rewritten, via replacing $r$ by $\sigma^{-1}(r)$, as

$$
r x=x \sigma^{-1}(r)-\delta \sigma^{-1}(r)=x \sigma^{-1}(r)-\tilde{\delta}(r),
$$


where $\tilde{\delta} \stackrel{\text { def. }}{=} \delta \sigma^{-1}=\tilde{\phi}(x)$ is a $\left(1, \sigma^{-1}\right)$-derivation. With the map $\tilde{\phi}$, the Ore extension $R[X ; D]$ can hence be alternatively defined with the commutation rule for $x \in X$ and $r \in R$ :

$$
r x=x \sigma^{-1}(r)-\tilde{\delta}(r)
$$

where $\tilde{\delta} \stackrel{\text { def. }}{=} \tilde{\phi}(x)$ is a $\left(1, \sigma^{-1}\right)$-derivation. So corresponding to any argument with map $\phi$, there is an argument with map $\tilde{\phi}$. The argument corresponding to the proof of Lemma 3 gives a proof of its left version, which we have stated above. We have to exploit this symmetry of $R[X ; D]$ frequently.

A ring $R$ is said to be right faithful if $a R=0$ implies $a=0$ for any $a \in R$. This is equivalent to $R$ being a dense right ideal of itself. For a right faithful ring $R$, the right Utumi quotient ring of $R$, denoted by $U_{r}(R)$, is the unique overring of $R$ such that for any $a \in U_{r}(R)$, there exists $\rho \varangle_{r t} R$ such that $a \rho \subseteq R$. Left faithful rings and left Utumi quotient rings are analogously defined. If $R$ is both right and left faithful, then the symmetric Utumi quotient ring of $R$, denoted by $U_{s}(R)$, is defined to be $U_{r}(R) \cap U_{\ell}(R)$ and can be characterized as the unique overring of $R$ such that for any $a \in U_{s}(R)$, there exist a dense right ideal $\rho$ and a dense left ideal $\lambda$ such that $a \rho \cup \lambda a \subseteq R$. More details can be found in [2, p. 51], [8, 14, 20].

The following important discovery due to D. Passman is true in the most general context. We learned this valuable result in [22] (p. 800).

Theorem 4 (Passman). If $R$ is right faithful, then $U_{r}(R[X ; D]) \supseteq U_{r}(R)$. Symmetrically, if $R$ is left faithful, then $U_{\ell}(R[X ; D]) \supseteq U_{\ell}(R)$. If $R$ is left and right faithful, then $U_{s}(R[X ; D]) \supseteq U_{s}(R)$.

Proof. For $a \in U_{r}(R), a \rho \subseteq R$ for some $\rho \boldsymbol{\triangleleft}_{r t} R$. By Lemma 3, $\rho[X ; D] \boldsymbol{\triangleleft}_{r t}$ $R[X ; D]$. Since $a \rho[X ; D] \subseteq R[X ; D], a \in U_{r}(R[X ; D])$ follows. So $U_{r}(R) \subseteq$ $U_{r}(R[X ; D])$. Symmetrically, we prove the left version $U_{\ell}(R) \subseteq U_{\ell}(R[X ; D])$. The last assertion follows from the equality $U_{s}(R)=U_{r}(R) \cap U_{\ell}(R)$.

\section{Proof of Theorem 1}

Let us fix an arbitrary linear order $<$ on $X$. The existence of such an order is assured by Zorn's Lemma. We extend $<$ to a linear order on words, also denoted by $<$, as follows: Given two words

$$
w \stackrel{\text { def. }}{=} y_{1} \cdots y_{m}, \quad v \stackrel{\text { def. }}{=} z_{1} \cdots z_{n}
$$

where $y_{i}, z_{j} \in X$, we postulate $w<v$ if $m \stackrel{\text { def. }}{=} \ln (w)<\operatorname{lh}(v) \stackrel{\text { def. }}{=} n$ or if $m=n$ and there exists $1 \leq k \leq m$ such that $y_{k}<z_{k}$ and such that $y_{i}=z_{i}$ for $1 \leq i<k$. That is, we order words first by length and then lexicographically for words of the same length.

In the expression (1) of $f \in R[X ; D]$ given in $\S 2$, we may assume $w_{0}>w_{1}>\cdots$. In this case, $w_{0}$, also denoted by $\hat{f}$, is called the leading word of $f$ and its coefficient $a_{0}$, also denoted by $\mathrm{c}(f)$, is called the leading coefficient of $f$. Write $\hat{f}=y_{1} \cdots y_{n}$, where $y_{i} \in X$, and suppose $\phi\left(y_{i}\right) \in \mathcal{L}_{\sigma_{i}}$. We define $\sigma_{f} \stackrel{\text { def. }}{=} \sigma_{1} \cdots \sigma_{n}$. Since $\hat{f} \in$ $R[X ; D]$, clearly $\sigma_{f}=\sigma_{\hat{f}}$ by definition. Throughout this section, $R$ is a domain. So $\widehat{f g}=\hat{f} \hat{g}, \mathrm{c}(f g)=\mathrm{c}(f) \sigma_{f}(\mathrm{c}(g))$ and $\sigma_{f g}=\sigma_{f} \sigma_{g}$ for nonzero $f, g \in R[X ; D]$.

Given $0 \neq \varphi \in U_{s}(R[X ; D])$, define

$$
\lambda_{\varphi} \stackrel{\text { def. }}{=}\{f \in R[X ; D]: f \varphi \in R[X ; D]\}, \quad \rho_{\varphi} \stackrel{\text { def. }}{=}\{g \in R[X ; D]: \varphi g \in R[X ; D]\} .
$$


Then $\lambda_{\varphi}$ is a dense left ideal of $R[X ; D]$ and $\rho_{\varphi}$ is a dense right ideal of $R[X ; D]$. For $f \in \lambda_{\varphi}$ and $g \in \rho_{\varphi}$, we have $f \varphi, \varphi g \in R[X ; D]$. Write

$$
\begin{aligned}
f & =\mathrm{c}(f) \hat{f}+\cdots, & g & =\mathrm{c}(g) \hat{g}+\cdots, \\
f \varphi & =\mathrm{c}(f \varphi) \widehat{f} \varphi+\cdots, & \varphi g & =\mathrm{c}(\varphi g) \widehat{\varphi g}+\cdots .
\end{aligned}
$$

Since $f(\varphi g)=(f \varphi) g$, comparing the leading terms of two sides yields

$$
\mathrm{c}(f) \sigma_{f}(\mathrm{c}(\varphi g)) \hat{f} \widehat{\varphi g}=\mathrm{c}(f \varphi) \sigma_{f \varphi}(\mathrm{c}(g)) \widehat{f \varphi} \hat{g} .
$$

So

$$
\hat{f} \widehat{\varphi g}=\widehat{f \varphi} \hat{g}
$$

and

$$
\mathrm{c}(f) \sigma_{f}(\mathrm{c}(\varphi g))=\mathrm{c}(f \varphi) \sigma_{f \varphi}(\mathrm{c}(g)) .
$$

Lemma 5. Assume that $R$ is a domain and $X$ has cardinality $\geq 2$. Given $0 \neq$ $\varphi \in U_{s}(R[X ; D])$, there exists a word $w$ in $X$ such that $\widehat{\varphi g}=w \hat{g}$ for all $g \in \rho_{\varphi}$ and such that $\widehat{f \varphi}=\hat{f} w$ for all $f \in \lambda_{\varphi}$.

Proof. Fix $0 \neq g \in \rho_{\varphi}$ arbitrarily. Assume that the leading word $\hat{g}$ begins with an indeterminate $y$. Since $|X| \geq 2$, there is $x \in X \backslash\{y\}$. Let $n$ be an integer $>\operatorname{lh}(\hat{g})$. By the left density of $\lambda_{\varphi}$, there exists $0 \neq h \in \lambda_{\varphi} \cap R[X ; D] x^{n}$. By $(\dagger)$,

$$
\hat{h} \widehat{\varphi g}=\widehat{h \varphi} \hat{g} \text {. }
$$

If $\ln (\hat{g})>\ln (\widehat{\varphi g})$, then the initial letter $y$ of $\hat{g}$ must occur in $\hat{h}$. But $\hat{h}$ ends with $x^{n}$, where $x \neq y$. So the initial letter $y$ of $\hat{g}$ must occur before the final segment $x^{n}$ of $\hat{h}$, implying

$$
\operatorname{lh}(\hat{g})>n+\operatorname{lh}(\widehat{\varphi g}) \geq n .
$$

But $n$ is chosen to be $>\operatorname{lh}(\hat{g})$, a contradiction. So $\operatorname{lh}(\hat{g}) \leq \operatorname{lh}(\widehat{\varphi g})$. Hence there exists a word $w_{g}$ in $X$ such that $\widehat{\varphi g}=w_{g} \hat{g}$. For any $0 \neq f \in \lambda_{\varphi},(\dagger)$ gives

$$
\widehat{f \varphi} \hat{g}=\hat{f} \widehat{\varphi g}=\hat{f} w_{g} \hat{g}
$$

implying $\widehat{f \varphi}=\hat{f} w_{g}$. This is true for any $0 \neq g \in \rho_{\varphi}$, since our $g \in \rho_{\varphi}$, though fixed, is arbitrary. For another $0 \neq g^{\prime} \in \rho_{\varphi}$, we also have $\widehat{f \varphi}=\hat{f} w_{g^{\prime}}$. Then $\hat{f} w_{g}=\widehat{f \varphi}=\hat{f} w_{g^{\prime}}$ implies $w_{g}=w_{g^{\prime}}$. So $w_{g}$ is independent of $0 \neq g \in \rho_{\varphi}$. Write $w$ for $w_{g}$. Then $\widehat{f \varphi}=\hat{f} w$ for all $f \in \lambda_{\varphi}$ and $\widehat{\varphi g}=w \hat{g}$ for all $g \in \rho_{\varphi}$, as asserted.

Lemma 6. Under the hypothesis of Lemma 5, there exist $a \in U_{s}(R)$ such that

$$
\mathrm{c}(f \varphi)=\mathrm{c}(f) \sigma_{f}(a) \quad \text { and } \quad \mathrm{c}(\varphi g)=a \sigma_{w}(\mathrm{c}(g)),
$$

for any nonzero $f \in \lambda_{\varphi}$ and $g \in \rho_{\varphi}$.

Proof. Since $R$ is a domain, so is $U_{s}(R[X ; D])$. So $0 \neq f \in \lambda_{\varphi}$ implies c $(f), \mathrm{c}(f \varphi) \in$ $R \backslash\{0\}$. Define the function $\theta$ on the set $\left\{\mathrm{c}(g): g \in \rho_{\varphi}\right\}$ by setting

$$
\theta(\mathrm{c}(g)) \stackrel{\text { def. }}{=} \mathrm{c}(\varphi g) \text {. }
$$

This is well-defined since $\mathrm{c}\left(g_{1}\right)=\mathrm{c}\left(g_{2}\right)$ implies $\mathrm{c}\left(\varphi g_{1}\right)=\mathrm{c}\left(\varphi g_{2}\right)$ by choosing $0 \neq$ $f \in \lambda_{\varphi}$ in $(\ddagger)$. With the function $\theta$, we rewrite $(\ddagger)$ as

$$
\mathrm{c}(f) \sigma_{f}(\theta(\mathrm{c}(g)))=\mathrm{c}(f \varphi) \sigma_{f \varphi}(\mathrm{c}(g)) .
$$


To be suggestive, we let

$$
\mathrm{c}\left(\rho_{\varphi}\right) \stackrel{\text { def. }}{=} \text { the additive group generated by the set }\left\{\mathrm{c}(g): g \in \rho_{\varphi}\right\} .
$$

Since $\rho_{\varphi}$ is a dense right ideal of $R[X ; D]$ and $R$ is a domain, $\mathrm{c}\left(\rho_{\varphi}\right)$ is a dense right ideal of $R$. We extend $\theta \mathbb{Z}$-linearly to $\mathrm{c}\left(\rho_{\varphi}\right)$ by setting

$$
\theta\left(\sum_{i} n_{i} \mathrm{c}\left(g_{i}\right)\right) \stackrel{\text { def. }}{=} \sum_{i} n_{i} \theta\left(\mathrm{c}\left(g_{i}\right)\right)
$$

for $g_{i} \in \rho_{\varphi}$ and $n_{i} \in \mathbb{Z}$. This is well-defined: If $\sum_{i} n_{i} \mathrm{c}\left(g_{i}\right)=0$, then for $f \in \lambda_{\varphi}$,

$$
\begin{aligned}
\mathrm{c}(f) \sigma_{f} & \left(\sum_{i} n_{i} \theta\left(\mathrm{c}\left(g_{i}\right)\right)\right)=\sum_{i} n_{i} \mathrm{c}(f) \sigma_{f}\left(\theta\left(\mathrm{c}\left(g_{i}\right)\right)\right) \\
& =\sum_{i} n_{i} \mathrm{c}(f \varphi) \sigma_{f \varphi}\left(\mathrm{c}\left(g_{i}\right)\right) \quad\left(\text { by }\left(\ddagger^{\prime}\right)\right) \\
& =\mathrm{c}(f \varphi) \sigma_{f \varphi}\left(\sum_{i} n_{i} \mathrm{c}\left(g_{i}\right)\right)=0 .
\end{aligned}
$$

Choose $f \neq 0$. Then $\mathrm{c}(f) \neq 0$. So $\sum_{i} n_{i} \theta\left(\mathrm{c}\left(g_{i}\right)\right)=0$ follows.

Observe that $\mathrm{c}(g) r=\mathrm{c}\left(g \sigma_{\hat{g}}^{-1}(r)\right)$ for $g \in R[X ; D]$ and $r \in R$. Given $f \in \lambda_{\varphi}$, $g \in \rho_{\varphi}$ and $r \in R$, we compute:

$$
\begin{aligned}
\mathrm{c}(f) \sigma_{f}(\theta(\mathrm{c}(g) r)) & =\mathrm{c}(f) \sigma_{f}\left(\theta\left(\mathrm{c}\left(g \sigma_{g}^{-1}(r)\right)\right)\right) \\
& =\mathrm{c}(f \varphi) \sigma_{f \varphi}\left(\mathrm{c}\left(g \sigma_{g}^{-1}(r)\right)\right) \quad\left(\mathrm{by}\left(\ddagger^{\prime}\right)\right) \\
& =\mathrm{c}(f \varphi) \sigma_{f \varphi}(\mathrm{c}(g) r) \\
& =\mathrm{c}(f \varphi) \sigma_{f \varphi}(\mathrm{c}(g)) \sigma_{f \varphi}(r) \\
& =\mathrm{c}(f) \sigma_{f}(\theta(\mathrm{c}(g))) \sigma_{f \varphi}(r) \quad\left(\mathrm{by}\left(\ddagger^{\prime}\right)\right) . \\
& =\mathrm{c}(f) \sigma_{f}\left(\theta(\mathrm{c}(g)) \sigma_{f}^{-1} \sigma_{f \varphi}(r)\right) .
\end{aligned}
$$

Pick $f \neq 0$. Then $\mathrm{c}(f) \neq 0$. The above yields

$$
\theta(\mathrm{c}(g) r)=\theta(\mathrm{c}(g)) \sigma_{f}^{-1} \sigma_{f \varphi}(r) .
$$

Let $w$ be the word asserted in Lemma 5 such that $\widehat{f \varphi}=\hat{f} w$ for $f \in \lambda_{\varphi}$. Then $\sigma_{f \varphi}=\sigma_{\widehat{f \varphi}}=\sigma_{\hat{f} w}=\sigma_{\hat{f}} \sigma_{w}=\sigma_{f} \sigma_{w}$. So $\sigma_{f}^{-1} \sigma_{f \varphi}=\sigma_{w}$. We hence have

$$
\theta(\mathrm{c}(g) r)=\theta(\mathrm{c}(g)) \sigma_{w}(r) .
$$

Appling $\sigma_{w}^{-1}$ to this identity, we have

$$
\sigma_{w}^{-1} \theta(\mathrm{c}(g) r)=\sigma_{w}^{-1} \theta(\mathrm{c}(g)) r .
$$

So $\sigma_{w}^{-1} \theta$ is a right $R$-module homomorphism defined on the dense right ideal $\mathrm{c}\left(\rho_{\varphi}\right)$. There thus exists $a^{\prime} \in U_{r}(R)$ such that $\sigma_{w}^{-1} \theta(\mathrm{c}(g))=a^{\prime} \mathrm{c}(g)$ for all $g \in \rho_{\varphi}$. Recall that $\theta(\mathrm{c}(g)) \stackrel{\text { def. }}{=} \mathrm{c}(\varphi g)$. Set $a \stackrel{\text { def. }}{=} \sigma_{w}\left(a^{\prime}\right) \in U_{r}(R)$. We have

$$
\mathrm{c}(\varphi g)=a \sigma_{w}(\mathrm{c}(g)) \quad \text { for } g \in \rho_{\varphi} .
$$


Plugging this into ( $\ddagger$ ) and using $\sigma_{f \varphi}=\sigma_{f} \sigma_{w}$, we have

$$
\begin{aligned}
\mathrm{c}(f \varphi) \sigma_{f \varphi}(\mathrm{c}(g)) & =\mathrm{c}(f) \sigma_{f}(\mathrm{c}(\varphi g)) \\
& =\mathrm{c}(f) \sigma_{f}\left(a \sigma_{w}(\mathrm{c}(g))\right) \quad\left(\text { since } \mathrm{c}(\varphi g)=a \sigma_{w}(\mathrm{c}(g))\right) \\
& =\mathrm{c}(f) \sigma_{f}(a) \sigma_{f} \sigma_{w}(\mathrm{c}(g)) \\
& =\mathrm{c}(f) \sigma_{f}(a) \sigma_{f \varphi}(\mathrm{c}(g)) \quad\left(\text { since } \sigma_{f \varphi}=\sigma_{f} \sigma_{w}\right) .
\end{aligned}
$$

Pick $0 \neq g \in \rho_{\varphi}$. Then $\mathrm{c}(g) \neq 0$ and hence $\sigma_{f \varphi}(\mathrm{c}(g)) \neq 0$. So $\mathrm{c}(f \varphi)=\mathrm{c}(f) \sigma_{f}(a)$ for $f \in \lambda_{\varphi}$. Let $\mathrm{c}^{\prime}\left(\lambda_{\varphi}\right)$ denote the additive subgroup generated by the set $\left\{\sigma_{f}^{-1}(\mathrm{c}(f))\right.$ : $\left.f \in \lambda_{\varphi}\right\}$. Then $\mathrm{c}^{\prime}\left(\lambda_{\varphi}\right) a \subseteq R$. If we write $f \in R[X ; D]$ in the form $\left(1^{\prime}\right)$, then the greatest word in this expression is still $\hat{f}$, but its coefficient (on the right side of $\hat{f}$ ) is $\sigma_{f}^{-1}(\mathrm{c}(f))$ instead of $\mathrm{c}(f)$. So by a symmetrical argument as above, $\mathrm{c}^{\prime}\left(\lambda_{\varphi}\right)$ forms a dense left ideal of $R$. It follows that $a \in U_{\ell}(R)$. So $a \in U_{s}(R)$, as asserted.

For $0 \neq f \in R[X ; D]$, we call $\mathrm{c}(f) \hat{f}$ the leading term of $f$ and denote it by $\operatorname{lt}(f)$. If $g \stackrel{\text { def. }}{=} f-\operatorname{lt}(f)$, then clearly $\hat{g}<\hat{f}$. By a subword of a word $y_{1} y_{2} \cdots y_{n}$, where $y_{i} \in X$, we mean the empty word 1 or a word in the form $y_{j_{1}} y_{j_{2}} \cdots y_{j_{m}}$, where $1 \leq j_{1}<j_{2}<\cdots<j_{m} \leq n$. The regular expression of $w r$ for any $r \in R$ clearly contains only subwords of $w$. We are now ready to give

Proof of Theorem 1. By Theorem 4, $U_{s}(R)[X ; D] \subseteq U_{s}(R[X ; D])$. So it suffices to prove the reverse inclusion. In view of $U_{s}(R[X ; D]) \supseteq U_{s}(R)$, it suffices to show

$$
U_{s}\left(U_{s}(R)[X ; D]\right)=U_{s}(R)[X ; D] .
$$

Replacing $R$ by $U_{s}(R)$, we may assume that $U_{s}(R)=R$ to start with. Given $0 \neq \varphi \in U_{s}(R[X ; D])$, we fix $0 \neq g \in R[X ; D]$ such that $\varphi g \in R[X ; D]$. By Lemmas 5 and 6 , there exist $a \in U_{s}(R)=R$ and a word $w$ in $X$ such that $\operatorname{lt}(\varphi g)=$ $a \sigma_{w}(\mathrm{c}(g)) w \hat{g}=\operatorname{lt}(a w g)$. Set $\varphi_{1} \stackrel{\text { def. }}{=} \varphi-a w$. Then $\varphi_{1} g=\varphi g-a w g \in R[X ; D]$. Since $\operatorname{lt}(\varphi g)=\operatorname{lt}(a w g)$, we have $\operatorname{lt}(\varphi g-a w g)<\operatorname{lt}(\varphi g)$, that is,

$$
\widehat{\varphi_{1} g}<\widehat{\varphi g} .
$$

Since $a w \in R[X ; D], \varphi_{1} \stackrel{\text { def. }}{=} \varphi-a w \in U_{s}(R[X ; D])$. By Lemmas 5 and 6 applied to $\varphi_{1}$, there exist a word $w_{1} \in \Omega$ and $a_{1} \in U_{s}(R)=R$ such that $\operatorname{lt}\left(\varphi_{1} g\right)=$ $a_{1} \sigma_{w_{1}}(\mathrm{c}(g)) w_{1} \hat{g}=\operatorname{lt}\left(a_{1} w_{1} g\right)$. Set $\varphi_{2} \stackrel{\text { def. }}{=} \varphi_{1}-a_{1} w_{1} \in U_{s}(R[X ; D])$. Analogously, $\varphi_{2} g \in R[X ; D]$ and $\widehat{\varphi_{2} g}<\widehat{\varphi_{1} g}$. We can again apply Lemmas [5] and 6 to $\varphi_{2}$. Continuing in this manner, we have

$$
\widehat{\varphi g}>\widehat{\varphi_{1} g}>\widehat{\varphi_{2} g}>\cdots \text {. }
$$

So all words in the regular expression of $\varphi_{i} g$ have length $\leq \operatorname{lh}(\widehat{\varphi g})$. Since $\varphi_{i+1} g=$ $\varphi_{i} g-a_{i} w_{i} g$, where $w_{i} \hat{g}=\widehat{\varphi_{i} g}$, indeterminates occurring in the regular expression of $\varphi_{i+1} g$ also occur in the regular expressions of $g, \varphi_{i} g$ and inductively in the regular expressions of $g, \varphi g$. We hence let $\Omega$ be the set of words of length $\leq \operatorname{lh}(\widehat{\varphi g)}$ and in the indeterminates occurring in the regular expressions of $g, \varphi g$. Then $\Omega$ contains all words involving regular expressions of $\varphi_{i} g$. Since $\Omega$ is finite, we must come to 
$\widehat{\varphi_{k+1} g}=0$ for some $k \geq 0$. This implies $\varphi_{k+1} g=0$ and hence $\varphi_{k+1}=0$. So

$$
\begin{aligned}
\varphi & =a w+\varphi_{1}=a w+a_{1} w_{1}+\varphi_{2}=\cdots \\
& =a w+a_{1} w_{1}+\cdots+a_{k} w_{k} \in R[X, D]=U_{s}(R)[X, D] .
\end{aligned}
$$

This completes the proof.

\section{REFERENCES}

[1] T. A. Andreeva, I. V. L'vov, and V. K. Kharchenko, The rings of quotients of free algebras, Algebra and Logic, 35(6)(1996), 366-370. MR1454681 (98h:16035)

[2] K.I. Beidar, W.S. Martindale III and A.V. Mikhalev, "Rings with Generalized Identities", Marcel Dekker, Inc., New York-Basel-Hong Kong, 1996. MR1368853 (97g:16035)

[3] J. Bergen and S. Montgomery, Ideals and quotients in crossed products of Hopf algebras, J. Algebra, 152 (1992), 374-396. MR1194308 (94a:16054)

[4] V. D. Burkov, On derivationally prime rings, Uspekhi Mat. Nauk., 35(5)(1980):219-220. Engl. Transl. Russian Math. Surveys, 35(5)(1980):253-254. MR.595145 (82f:16002)

[5] W. Cortes and M. Ferrero, Partial skew polynomial rings: Prime and maximal ideals, Commun. Algebra, 35(4) (2007), 1183-1199. MR2313659(2008b:16038)

[6] C. L. Chuang, T. K. Lee, C. K. Liu, and Y. T. Tsai, Higher derivations of Ore extensions, Israel J. Math, 175 (2010), 157-178.

[7] C. L. Chuang and Y. T. Tsai, On the structure of semi-invariant polynomials in Ore extensions, J. Algebra, 322 (2009), 2464-2491. MR.2553690

[8] Carl Clifton Faith, "Lectures on Injective Modules and Quotient Rings", Volume 49 of Lecture Notes in Mathematics, Springer-Verlag, Berlin-New York, 1967. MR0227206 (37:2791)

[9] E. Formanek, Maximal quotient rings of group rings, Pacific J. Math., 53(1) (1974), 109-116. MR0382325 (52:3210)

[10] K. R. Goodearl, Prime ideals in skew polynomial rings and quantized Weyl algebras, J. Algebra, 150 (1992), 324-377. MR1176901 (93h:16051)

[11] P. Grzeszczuk, A. Leroy and J. Matczuk, Artinian property of constants of algebraic q-skew derivations, Israel J. Math., 121 (2001), 265-284. MR.1818391 (2002b:16050)

[12] T.Y. Lam, A. Leroy, K.H. Leung and J. Matczuk, Invariant and semi-invariant polynomials in skew polynomial rings, Israel Mathematics Conference Proceedings, 1 (1989), 247-261. MR.1029317 (90k:16004)

[13] T. Y. Lam and A. Leroy, "Homomorphisms between Ore Extensions", Contemporary Math., vol. 124, Amer. Math. Soc., Providence, RI, 1992, 83-110. MR1144030 (93b:16052)

[14] J. Lambek, "Lectures on Rings and Modules", second edition, Chelsea, New York, 1976. MR0419493 (54:7514)

[15] S. Lanning, The maximal symmetric ring of quotients, J. Algebra, 179 (1996), 47-91. MR1367841 (96m:16040)

[16] A. Leroy and J. Matczuk, The extended centroid and X-inner automorphisms of Ore extensions, J. Algebra, 145 (1992), 143-177. MR.1144664 (93b:16053)

[17] A. Leroy, J. Matczuk and E. Puczylowski, Quasi-duo skew polynomial rings, J. Pure and Applied Algebra, 212(8) (2008), 1951-1959. MR2414695 (2009c:16082)

[18] K. Louden, Maximal quotient rings of ring extensions, Pacific J. Math., 62(2) (1976), 489496. MR0407059 (53:10842)

[19] D. S. Passman, Computing the symmetric ring of quotients, J. Algebra, 105 (1987), 207-235. MR 871754 (88b:16065)

[20] D. S. Passman, "A Course in Ring Theory", Wadsworth \& Brooks/Advanced Books \& Software, Pacific Grove, California, 1991. MR.1096302 (91m:16001)

[21] J. D. Rosen and M. P. Rosen, Extended centroids of skew polynomial rings, Canad. Math. Bull., 28(1) (1985), 67-76. MR778263 (86d:16005)

[22] J. D. Rosen and M. P. Rosen, Ideals and quotient rings of skew polynomial rings, Commun. Algebra, 21(3) (1993), 799-823. MR 1204755 (94a:16053)

[23] J. D. Rosen and M. P. Rosen, The Martindale ring of quotients of a skew polynomial ring of automorphism type, Commun. Algebra, 21(11) (1993), 4051-4063. MR 1238143 (94g:16038)

[24] M. Smith, Group algebras, J. Algebra, 18(1971), 477-499. MR0277625 (43:3358) 
[25] Y.-T. Tsai and C.-L. Chuang, Quotient rings of Ore extensions with more than one indeterminate, Commun. Algebra, 36(10) (2008), 3608-3615. MR2458396 (2009k:16054)

[26] Y.-T. Tsai, T.-Y. Wu, and C.-L. Chuang, Jacobson radicals of Ore extensions of derivation type, Commun. Algebra, 35(03) (2007), 975-982. MR.2305244 (2007m:16045)

Department of Mathematics, National Taiwan University, Taipei 106, Taiwan

E-mail address: chuang@math.ntu.edu.tw

Department of Applied Mathematics, Tatung University, Taipei 104, Taiwan

E-mail address: yttsai@ttu.edu.tw 